\title{
Urethral injury in laparoscopic-assisted abdominoperineal resection
}

\author{
Laurel Stitt, MD, CCFP; Francisco Avila Flores, MD, FRCPC; Sonny S. Dhalla, MD, FRSCS, FACS
}

University of Manitoba, Brandon Regional Health Centre, Brandon, MB, Canada

Cite as: Can Urol Assoc J 2015;9(11-12):E900-2. http://dx.doi.org/10.5489/cuai.3259

Published online December 14, 2015.

\section{Abstract}

We present a 71-year-old man who underwent laparoscopic-assisted abdominoperineal resection for recurrence of rectal cancer, which was complicated by a urethral injury. Traumatic urinary catheter insertion was ruled out as an alternative etiology. This case highlights the importance of recognizing urethral injury as a possible complication of laparoscopic-assisted abdominoperineal resection surgery.

\section{Introduction}

Urethral injuries are a rare complication of colorectal/ perineal surgery; most commonly, they are associated with traumatic urinary catheter placement preoperatively. ${ }^{1}$ It is expected that the perineal part of the surgery would have a higher incidence of urethral injury, which likely would be recognized intraoperatively or immediately postoperatively. Our PubMed literature search yields paucity of recent data on the incidence of urethral injuries in colorectal surgeries..$^{2-4}$ When considering risk factors for any urological injury, presence of malignancy, metastatic disease, and rectal procedures have been associated with increased rates of bladder and ureteric injuries. ${ }^{2}$ In our review of the literature, we found two articles reporting urethral injuries. ${ }^{3,4}$ From the 579 cases described in the more recent paper, ${ }^{3}$ two cases of urethral injury were identified, but no data regarding mechanism or treatment was reported. A previous older report ${ }^{4}$ described two cases (1.8\% incidence) of urethral injuries, both recognized intraoperatively.

\section{Case report}

A 71-year-old man underwent laparoscopic-assisted abdominoperineal resection (LAAPR) for recurrence of rectal carcinoma. Initially diagnosed with pT3 N1c M0 disease, he underwent low anterior resection with protective ileostomy one year previously, and declined adjuvant chemoradiation. During followup colonoscopy, he was found to have recurrence at his anastamosis, and underwent neoadjuvant chemoradiation, with a total of 5040 cGy in 28 fractions administered to the pelvis and tumour bed. This was followed by LAAPR, as well as takedown of protective loop ileostomy, placement of sigmoid end permanent colostomy, and repair of parastomal hernia. No course corrections were employed. Dissection around the anal sphincter muscle up to the coccyx was done using electrocautery. Adhesions between the rectum and lower pelvis were divided using a Harmonic scalpel to gain access to the pelvic cavity. The remainder of the perineal dissection was completed using the Harmonic scalpel. The case proceeded without intraoperative complications; blood loss was approximately $500 \mathrm{~mL}$. A Jackson-Pratt (JP) drain was brought out via the abdomen.

The patient's postoperative course was complicated by an ileus, briefly requiring total parenteral nutrition. Urinary ascites was not considered as an etiology at that time. Epidural and urinary catheters were discontinued on the third and fourth postoperative days (POD), respectively. There was ongoing JP drainage of more than $50 \mathrm{~mL}$ per day. He was discharged home on POD 14 with home care services to assist with JP drain management.

Drain output persisted, and on POD 17 the fluid was analyzed for creatinine. This result was $6.7 \mu \mathrm{mol} / \mathrm{L}$, indicating that the drainage fluid was not consistent with urine. The source of the fluid was, therefore, thought to be a seroma.

One week later, the patient reported the observation that the drain output would increase during voiding. A cystourethrogram demonstrated an approximately $9 \mathrm{~mm}$ defect of the inferior aspect of the posterior urethra that allowed extravasation of contrast. A tract extended from the urethral defect to the presacral space where the JP drain was present (Figs. 1 and 2).

The patient was managed conservatively with an indwelling urinary catheter, which was inserted without difficulty. The JP drain output decreased, and 14 days later the patient reported inadvertent removal of his drain. As there was no 


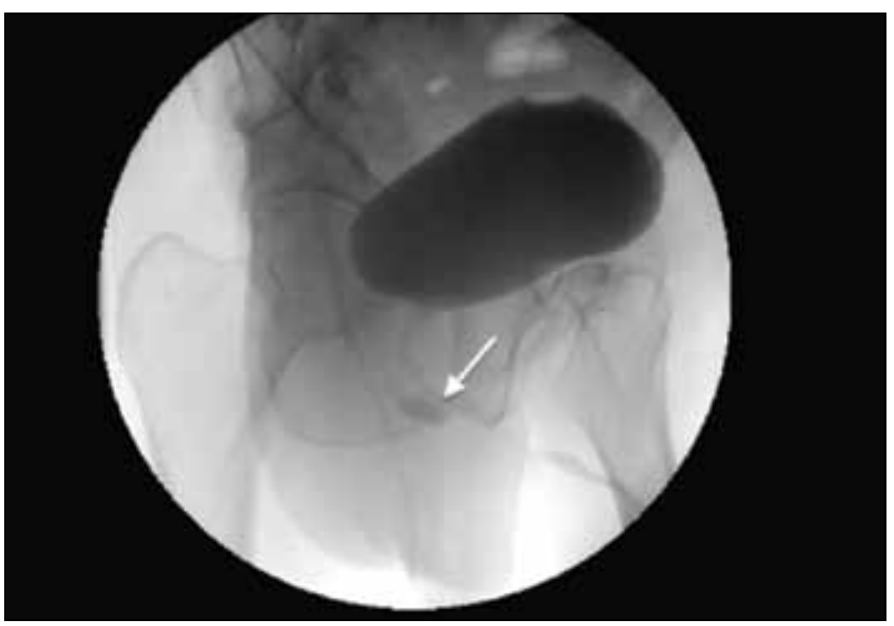

Fig. 1. Cystourethrogram demonstrating a tract arising from the posterior urethra.

evidence of ongoing intra-abdominal collection, the drain was not replaced. A repeat cystourethrogram five weeks after urinary catheter insertion showed a small residual collection in the posterior membranous urethra. A subsequent third cystourethrogram six weeks later demonstrated complete resolution of the urethral leak, and the urinary catheter was removed. The patient was able to void without complications.

In our facility, preoperative urinary catheter insertion is typically performed by the operating room nursing staff. The patient's operative records show no complications of urinary catheter insertion, and there is no documentation of hematuria intra-operatively. However, the patient's immediate postoperative records indicate the presence of mild hematuria, which resolved spontaneously later that day. At the time, this finding was attributed to use of a bladder retractor intraoperatively.

\section{Discussion}

Urethral injury in colorectal/perineal surgery is rare and minimal recent data exists on the topic. In one recent retrospective study of 579 patients who underwent laparoscopic surgery for rectosigmoid and rectal cancer, there were only two cases of intraoperative urethral injury. ${ }^{3}$ It is unknown whether these injuries were the result of traumatic urinary catheter placement as opposed to intraoperative complications. The study reported neither the type of surgery performed for these two cases of urethral injury, nor the treatment.

An older retrospective study from 1976 examined 111 patients who had undergone abdominoperineal resection for malignancies of the rectum or anal canal. ${ }^{4}$ Two cases of intraoperative urethral injury were described; both cases were complications of surgery and were recognized intraoperatively. One case was managed with primary repair, the other managed with insertion of long-term urinary catheter.

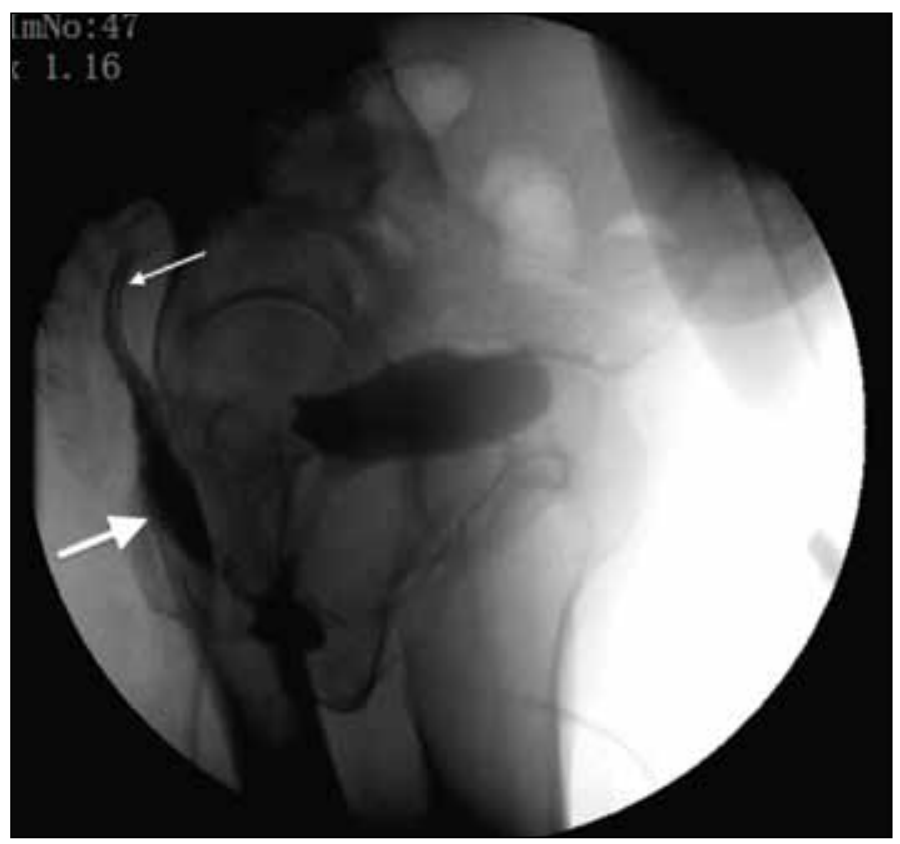

Fig. 2. Note the JP drain with its tip situated in the presacral region (small arrow), and the fluid collecting adjacent to the drain (large arrow).

Generally, it is anticipated that drainage fluid from a urinary leak would have a measured creatinine level greater than serum creatinine. In our case, this measurement failed to help diagnose urinary leakage, likely due to the intermittent nature of the urethral leak correlated with the patient's voiding.

Etiology of our case remains speculative. This patient is a re-do of an anterior resection, anticipating scarring, with neoadjuvant radiation therapy and associated potential for non-healing. Since an experienced perineal surgeon did not recognize a urethral injury intraoperatively, it is possible that electrocautery use during resection of tissues surrounding the urethra could have resulted in collateral thermal damage. This would weaken the wall of the urethra, resulting in ischemia and leading to disruption of the wall and subsequent leakage. The patient did not have any signs of infection of the perineum, and there is no history of traumatic urinary catheter insertion.

In summary, urethral injury is a rare but important complication of colorectal/perineal surgery. Clinicians should be aware of the potential for urethral injury. Certain features of the patient history and presenting symptoms may be crucial in making a timely diagnosis.

Competing interests: The authors declare no competing financial or personal interests.

Acknowledgments: We gratefully acknowledge Dr. David Lysack for his contribution to this case report. 
Stitt et al.

This paper has been peer-reviewed.

\section{References}

1. Delacroix SE, Winters JC. Urinary tract injuries: recognition and management. Clin Colon Rectal Surg 2010;23:104-12. http://dx.doi.org/10.1055/s-0030-1254297
2. Sawkar HP, Kim DY, Thum DJ, et al. Frequency of lower urinary tract injury after gastroin-testinal surgery in the nationwide inpatient sample database. Am Surg 2014;80: 1216-21.

3. $\mathrm{Ng} \mathrm{KH}, \mathrm{Ng} \mathrm{DC}$, Cheung $\mathrm{HY}$ et al. Laparoscopic resection for rectal cancers: Lessons learned from 579 cases. Ann Surg 2009;249: 82-6. http://dx.doi.org/10.1097/SLA.0b013e31818e418a

4. Andersson A, Bergdahl L. Urologic complications following abdominoperineal resection of the rectum. Arch Surg 1976;111:969-71. http://dx.doi.org/10.1001/archsurg.1976.01360270041007

Correspondence: Dr. Laurel Stitt, Brandon Medical Arts Clinic, Brandon, MB; stitt.laurel@gmail.com 